\title{
Stepwise Synthesis of Oligopeptides with $N$-Carboxy $\alpha$-Amino Acid Anhydrides. V. Synthesis and NMR Study of Oligopeptides with Some Sequences of L-Alanine and L-Valine
}

\author{
Ryoichi Katakai and Masanao OYA, \\ Department of Industrial Chemistry, College of Technology, \\ University of Gunma, Kiryu, Gunma, Japan. \\ Keikichi UnO and Yoshio IwaKURA \\ Department of Synthetic Chemistry, Faculty of Engineering, \\ The University of Tokyo, Bunkyo-ku, Tokyo, Japan.
}

(Received March 4, 1972)

\begin{abstract}
Oligopeptides with the sequences of alternating L-alanine and L-valine and of a short block of the two amino acids were synthesized in high yields by the NCA method in a heterogeneous system of acetonitrile-water. The NMR spectra of methyl protons in the side chains of the oligopeptides in TFA showed a clear difference in chemical shifts resulting from the sequential position, $C$-terminal, internal and $N$ terminal, of the amino acid residues in the oligopeptides. Deshielding effects of terminal carboxyl and ammonium groups as compared with that of peptide linkage was estimated from the spectra of the oligopeptides.

KEY WORDS Oligopeptide / Synthesis / NMR / Chemical Shift / Deshielding Effect / L-Alanine / L-Valine /
\end{abstract}

Extensive studies have been made on naturally occurring oligopeptides and polypeptides with respects to the synthetic method and the biological activity. However there are few studies on oligopeptides as a model compound for comformational studies. ${ }^{1-7}$ In our opinion much more attention should be paid to the preparation of oligopeptides as model compounds for polypeptides.

This paper deals with the synthesis of oligopeptides having a sequence of L-valyl-L-alanyland L-valyl-L-valyl-L-alanyl-L-alanyl- residues that are model compounds of alternating and short block copolypeptides of alanine and valine, and with the NMR study of these peptides in trifluoroacetic acid (TFA).

\section{RESULTS AND DISCUSSION}

\section{Synthesis of Oligopeptides}

In the case of conventional methods for synthesizing oligopeptides, many reaction steps are needed for protection of the amino acid in coupling reaction and removal of the protecting groups from the resulting peptide. Oligopeptides, therefore, are not obtained easily in high yield even in the case of amino acids having nonpolar side chains. In the present study NCA's were used for the synthesis of oligopeptides in a heterogeneous system of acetonitrile-water without homopolymerization of NCA. ${ }^{8-10}$ In this method direct acylation of an amino acid or a peptide by NCA made the synthetic process fast and easy and peptides were obtained in high yield.

Oligopeptides having a sequence of alternating L-alanine and L-valine residue (I) were synthesized by addition of a solution of $\mathrm{L}$-alanine NCA in acetonitrile into the heterogeneous mixture of acetonitrile and water containing sodium salt of L-valine as a starting amino acid and sodium carbonate at $-10^{\circ} \mathrm{C}$, followed by sequential additions of L-valine NCA and Lalanine NCA without isolation of intermediate peptides. 
Stepwise Synthesis of Oligopeptides with NCA. V.

Table I. Results of syntheses of oligopeptides

\begin{tabular}{|c|c|c|c|c|c|c|c|c|c|}
\hline \multirow{3}{*}{ Peptide } & \multirow{3}{*}{\multicolumn{2}{|c|}{ Yield, $\% \mathrm{Rf}^{\mathrm{a}}$}} & \multirow{3}{*}[\alpha]{$_{\mathrm{D}}$ in TFA } & \multicolumn{6}{|c|}{ Elemental analysis } \\
\hline & & & & \multicolumn{3}{|c|}{ Calcd } & \multicolumn{3}{|c|}{ Found } \\
\hline & & & & $\mathrm{C}, \%$ & $\mathrm{H}, \%$ & $\mathrm{~N}, \%$ & $\mathrm{C}, \%$ & $\mathrm{H}, \%$ & $\mathrm{~N}, \%$ \\
\hline H-Val-Ala-Val-OH & 94 & 0.48 & -26.5 (c 1.7$)$ & 54.32 & 8.79 & 14.62 & 54.43 & 8.83 & 14.54 \\
\hline H-Ala-Val-Ala-Val-OH & 86 & 0.44 & $-60.6(\mathrm{c} 1.3)$ & 53.60 & 8.45 & 15.63 & 53.56 & 8.55 & 15.58 \\
\hline H-Val-Ala-Val-Ala-Val-OH & 84 & 0.39 & $-71.3(\mathrm{c} 2.3)$ & 55.11 & 8.61 & 15.31 & 55.23 & 8.74 & 15.18 \\
\hline H-Ala-Val-Ala-Val-Ala-Val-OH & 81 & 0.36 & $-92.1(\mathrm{c} 2.2)$ & 54.52 & 8.40 & 15.90 & 54.38 & 8.52 & 15.96 \\
\hline H-Val-Val-Ala-Ala-OH & 82 & 0.47 & $-87.7(\mathrm{c} 2.3)$ & 53.60 & 8.45 & 15.63 & 53.47 & 8.51 & 15.65 \\
\hline H-Ala-Ala-Val-Val-Ala-Ala-OH & 77 & 0.25 & $-110.2(\mathrm{c} 2.6)$ & 52.77 & 8.07 & 16.79 & 52.62 & 8.15 & 16.63 \\
\hline $\begin{array}{l}\text { Val-Val-Ala-Ala-Val-Val- } \\
\text { Ala-Ala-OH }\end{array}$ & 70 & 0.21 & -117.2 (c 3.2) & 55.78 & 8.50 & 16.27 & 55.86 & 8.58 & 16.18 \\
\hline
\end{tabular}

a $\mathbf{R f}$ were measured on silica gel thin-layer with butanol-acetic acid-water-(4:1:1) as a developer.

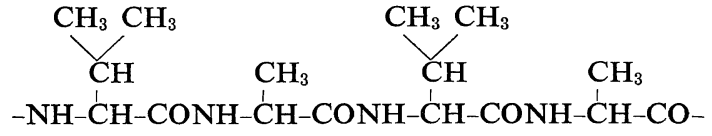

( I )

The short-block-type peptides (II) were obtained by sequential additions of L-alanine NCA and L-valine NCA into L-alanine as a starting amino acid.

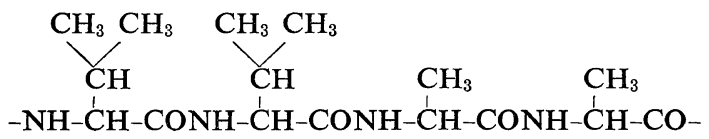

(II)

The NCA method for preparing Oligopeptide in the heterogeneous system of acetonitrilewater has the advantages that the reaction of NCA with an amino acid and a peptide proceeds quantitatively and that side reactions as polymerization and hydrolysis of the $\mathrm{NCA}^{8}$ and hydantoic acid formation ${ }^{10}$ practically do not occur. In the present syntheses, the purity of the intermediate peptides was ckecked at the each step after the reaction and minor products could be removed by recrystallization of the final product. The resulting oligopeptides were chromatographically pure. Results of syntheses are summarized in Table I.

This NCA method may be especially useful for the preparation of peptides as model compounds for the study of polypeptides.

\section{NMR Spectra of Oligopeptides}

Oligopeptides having nonpolar side chains were hardly soluble in many common solvents such as water, alcohols, dimethylformamide, and dimethylsulfoxide; and were insoluble in hydrocarbons and ethers. Trifluoroacetic acid (TFA) is known to be a good solvent for peptides and allows the polypeptide to assume a random coil structure. In the present study, therefore, TFA was used as a solvent for NMR studies in order to avoid the complexity of analysis of the NMR spectra due to any secondary structure. Poly(L-alanine) has a random coil structure in TFA and poly(Lvaline) does not have an $\alpha$-helical structure owing to the steric hindrance of the side chain. Seen from the relatively low degree of polymerization of the present oligopeptides consisting of L-alanine and L-valine, a secondary structure

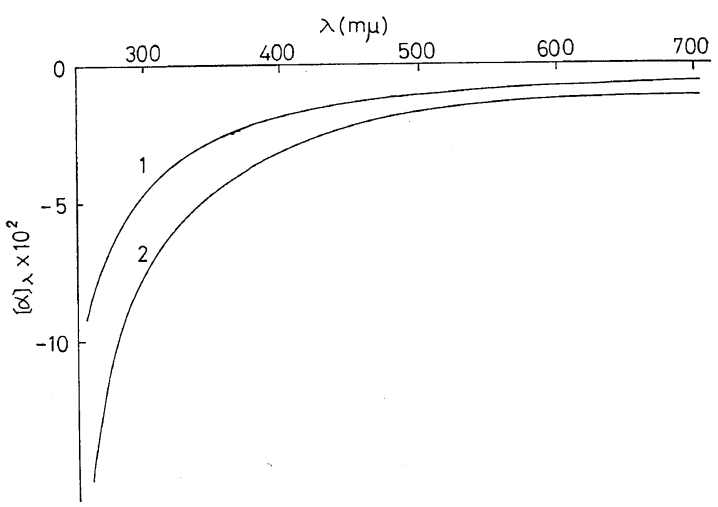

Figure 1. ORD curves of oligopeptides: 1, Lvalyl-L-alanyl-L-valyl-L-alanyl-L-varine in TFATFE ( $3: 10$ by volume); 2, L-alanyl-L-valyl-Lalanyl-L-valyl-L-alanyl-L-valine in TFA-TFE $(5: 5)$. 
such as an $\alpha$-helix cannot occur in TFA. Experimentally the random coil structure was proved through optical rotatory dispersion (ORD) measurements in TFA - trifluoroethanol, which showed simple dispersion curves for the oligopeptides (Figure 1).

Figure 2 shows the NMR spectrum of Lvalyl-L-alanyl-L-valine in TFA. There were resonance peaks at $8.86,8.41,7.62,5.65,5.26$, 2.64, and $2.10 \tau$. The triplet peak at $8.86 \tau$ was assigned to the methyl protons of valine residues $^{12}$ and the doublet peak at $8.41 \tau$ was to the methyl protons of alanine that couples with $\alpha$-methyne of the amino acid appearing at $5.26 \tau$. The multiplet peak at $7.62 \tau$ was assigned to $\beta$-methyne proton of valine that couples with the methyl and $\alpha$-methyne protons of valine appearing at $5.65 \tau$. Protons of amino group that is protonated in TFA were observed at $2.64 \tau$ and the area of the peak was three times as that of the protons in peptide bond at $2.10 \tau$. All other oligomers having a sequence of alternating two amino acid residues showed almost the same NMR spectra except for the methyl protons of the side chains.

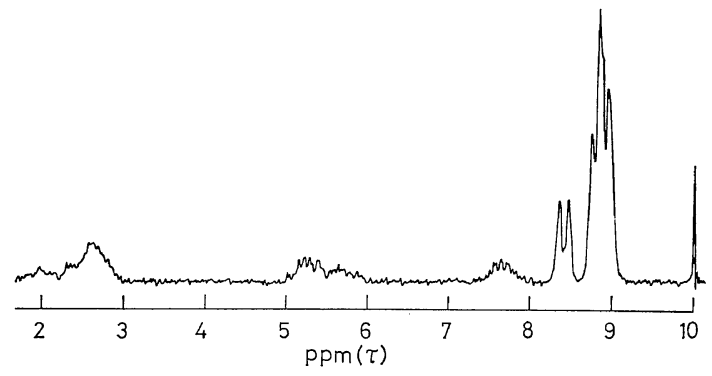

Figure 2. NMR spectrum of L-valyl-L-alanyl-Lvaline in TFA at room temperature.

The methyl protons of the side chains showed clearly different chemical shifts resulting from the difference in the sequential position of amino acid in the oligopeptides. The partial spectra covering the methyl proton region are shown in Figure 3. In these spectra resonance peaks appearing at $9.1-8.6 \tau$ are those of the methyl protons of valine residues, and those at 8.6$8.1 \tau$ are those of alanine residues. The dipeptide, L-alanyl-L-valine, showed a doublet peak, $J=7 \mathrm{cps}$, at $8.19 \tau$ for methyl propons
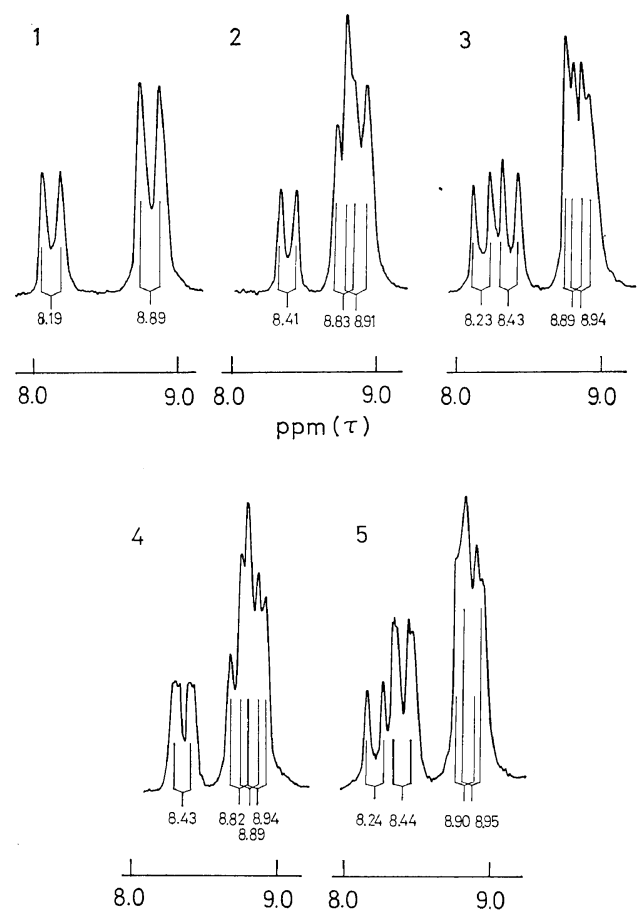

Figure 3. The partial NMR spectra covering the peaks of methyl protons in the oligopeptides consisling of L:alanyl-L-valyl-residue 1, L-alanylL-valine; 2, L-valyl-L-alanyl-L-valine; 3, L-alanylL-valyl-L-alanyl-L-valine; 4 , L-valyl-L-alanyl-Lvalyl-L-alanyl-L-valine; $5, \quad$ L-alanyl-L-valyl-Lalanyl-L-valyl-L-ananyl-L-valine.

of N-terminal alanine and a doublet at $8.89 \tau$, $J=6 \mathrm{cps}$, for those of the $C$-terminal valine residure. The tripeptide, L-valyl-L-alanyl-Lvaline, showed peaks at $8.41,8.83$, and $8.91 \tau$. The doublet peak at $8.41 \tau$ is that of the methyl resonance for the internal alanine moiety; this shifted from $8.19 \tau$ for the N-terminhal alanine in the dipeptide, and a new doublet peak was seen at $8.83 \tau$ for the $\mathrm{N}$-terminal valine, which overlapped with the doublet $C$-terminal valine peak at $8.91 \tau$ to show a triplet peak. The peak for $\mathrm{N}$-terminal valine at $8.83 \tau$ disappeared, and a quartet peak for two valine moieties was seen at $8.92 \tau$ which is divided to two doublet peaks at 8.89 and $8.94 \tau$ for the tetrapeptide, L-alanylL-valyl-L-alanyl-L-valine. That the resonance peaks for the methyl protons of the amino acid residue at the internal position in oligopeptide occur at a higher $\tau$ value than those at the $C$ - 
Stepwise Synthesis of Oligopeptides with NCA. V.

terminal position in the oligopeptide was demonstrated for the methyl groups of alanine residues in the octapeptide of L-valyl-L-valyl-Lalanyl-L-alanyl-L-valyl-L-valyl-L-alanyl-L-alanine (Figure 4(3)). The peaks of higher $\tau$ values showed by the methyl protons of alanine in the octapeptide had a peak area three times as great as the peaks of the lower $\tau$ value, thus the former can be assigned to the internal alanine residues and the latter to those of C-terminal. As this may be true for the methyl protons of valine residues and moreover the peaks for the $C$-terminal valine in the lower oligopeptides were observed at $8.89-9.91 \tau$, the observed peaks at $8.94 \tau$ for the tetrapeptide can be assigned to the internal valine residue and those at $8.89 \tau$ to the $C$-terminal. The peptide, $\mathrm{L}-$ valyl-L-alanyl-L-valyl-L-alanyl-L-valine, showed the peaks for three different species of valine, $N$-terminal, internal and $C$-terminal and only one peak due to internal alanine residues. The doublet peak of alanine was broadened, so that there may be a slight differences may be undetectable at $60 \mathrm{MHz}$. This difference in the chemical shifts of the internal alanine residues, however, may be negligible for a first-order approximation in the estimation of deshielding effect for peptide linkage as made below. The hexapeptide showed two types of valine residues, $C$-terminal and two internal, and also two types of alanine residues, $N$-terminal and two internal. Assignments for the peaks are summarized in Table II.

Table II. NMR chemical shifts of methyl protons of $\mathrm{L}$-alanine and $\mathrm{L}$-valine in the oligopeptides consisting of L-alanyl-L-valyl-residue

\begin{tabular}{rrrrrr}
\hline \multicolumn{6}{c}{ Chemical shift } \\
$\mathrm{H}-\mathrm{Ala}$ - Val $-\mathrm{Ala}$ - Val - Ala - Val $-\mathrm{OH}$ \\
\hline 8.24 & 8.95 & 8.44 & 8.95 & 8.44 & 8.90 \\
& 8.82 & 8.43 & 8.94 & 8.43 & 8.89 \\
& & 8.23 & 8.94 & 8.43 & 8.89 \\
& & & 8.83 & 8.41 & 8.91 \\
& & & & 8.19 & 8.89 \\
\hline
\end{tabular}

The partial NMR spectra of oligopeptides having a sequence of short blocks of L-alanine and L-valine residues are shown in Figure 4. The tetrapeptide, L-valyl-L-valyl-L-alanyl-L-
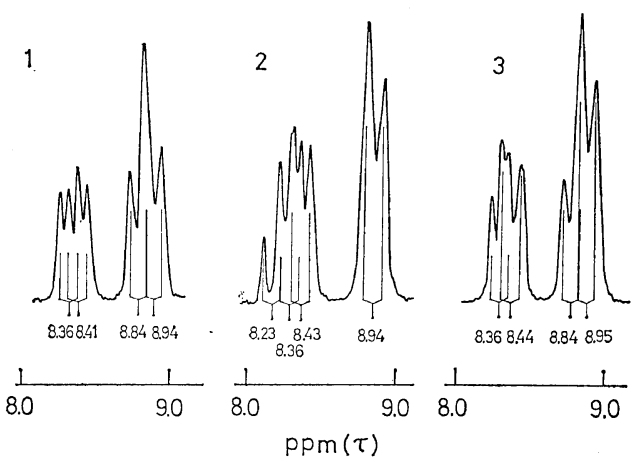

Figure 4. The partial NMR spectra covering the peaks of methyl protons in the oligopeptides consisting of L-Valyl-L-valyl-L-alanyl-L-alanylresidue: 1, L-valyl-L-valyl-L-alanyl-L-alanine; 2, L-alanyl-L-alanyl-L-valyl-L-valyl-L-alanyl-L-alanine; 3, L-valyl-L-valyl-L-alanyl-L-alanyl-L-valyl-L-valylL-alanyl-L-alanine.

alanine, showed peaks at $8.84 \tau$ for the methyl protons of $N$-terminal valine, at $8.94 \tau$ for those of internal valine, at $8.36 \tau$ for $C$-terminal alanine moieties. The hexapeptide and octapeptide showed NMR spectra having the same peak positions as the tetrapeptide and the alternating cooligopeptides shown above, which have $\tau$ values of 8.94 for internal and of 8.84 for N-terminal valines, and $8.41-8.44,8.36$ and 8.23 for internal, $C$-terminal, and $N$-terminal alanine moieties, respectively. Assignment of the NMR peaks of the oligopeptides having short block sequences are given in Table III.

Table III. NMR chemical shift of methyl protons of $\mathrm{L}$-alanine and $\mathrm{L}$-valine in the oligopeptides consisting of L-valyl-L-valyl-L-alanylL-alanyl-residue

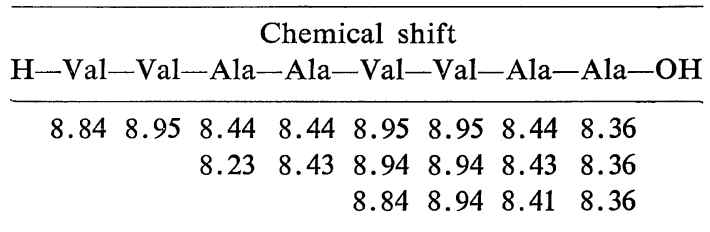

In TFA the oligopeptides showed the same peak position for methyl protons in the same amino acid residue which had the same sequential position in the oligopeptides. From Tables II and III, the NMR spectra of the methyl protons are summarized. It is seen that 
$\mathrm{N}$-terminal alanine appears at $8.23 \tau$, internal at $8.41-8.44$ and $C$-terminal at $8.36 \tau$. The $N$ terminal, internal, and $C$-terminal valine moieties gave peaks at $8.84,8.99$, and $8.96 \tau$, respectively. These results may suggest that the sequence of small oligopeptides (tri- and di-peptides) containing alanine and valine can be determined from the NMR chemical shifts for methyl protons in the side chain in TFA as well as in aqueous solution. ${ }^{11}$

The difference in $\tau$ value for the same amino acid residue reflects the difference in deshielding effect arising from the electron-withdrawing effect of the charged ammonium group and the dipoles of the carboxyl group and peptide linkage. The strength of the effect decreased in the order of $\mathrm{NH}_{3}{ }^{+}>\mathrm{COOH}>\mathrm{CONH}$. These effects can be estimated from above results: the increasing effect of the terminal caboxyl group for alanine in $\tau$ value, $\Delta_{\mathrm{AC}}$, is the difference between $C$-terminal resonance peak position and internal one, so

$$
\Delta_{\mathrm{AC}}=8.36-8.44=-0.08 \tau,
$$

and the effect of the terminal $\mathrm{NH}_{3}{ }^{+}, \Delta_{\mathrm{AN}}$, is

$$
\Delta_{\mathrm{AN}}=8.23-8.44=-0.21 \tau,
$$

For valine, the effect of carboxyl group, $\Delta_{\mathrm{VC}}$, is

$$
\Delta_{\mathrm{VC}}=8.90-8.95=-0.05 \tau,
$$

and also the effect of the terminal $\mathrm{NH}_{3}{ }^{+}, \Delta_{\mathrm{VN}}$, is

$$
\Delta_{\mathrm{VN}}=0.84-8.95=-0.11 \tau .
$$

The difference in $\tau$ for two amino acids, $\Delta_{\mathrm{AN}}$ and $\Delta_{\mathrm{VN}}$, and also $\Delta_{\mathrm{AC}}$ and $\Delta_{\mathrm{VC}}$, shows that the effect is shielded by the presence of methyn group in the side chain. The values of $\tau$ for $\gamma$-methyl protons of valine, theorefore, are smaller than those for $\beta$-methyl protons of alanine.

An amino acid has both of the effects estimated above. Therefore alanine must have a resonance peak for methyl protons at $8.15 \tau$, because

$$
8.44-0.08-0.21=8.15 \tau,
$$

and valine must have a resonance at $8.79 \tau$, because

$$
8.95-0.05-0.11=8.79 \tau .
$$

These values expected from the above estimates are quite consistent with the measurements in TFA by Bovey and Tiers: ${ }^{12} 8.15 \tau$ for methyl protons of DL-alanine and $8.81 \tau$ for those of DL-valine. One is able to estimate the peak positions of an amino acid from the NMR spectra of the oligopeptides containing the amino acid. The estimation may be acceptable for other oligopeptides that have no secondary structure.

\section{EXPERIMENTAL}

General Procedure for Synthesis of Oligopeptides ${ }^{8}$

A sodium salt of an amino acid, $0.01 \mathrm{~mol}$, was dissolved $50 \mathrm{~m} l$ of aqueous solution of $0.2-N$ sodium hydroxide and sodium carbonate, 0.01 mol, and $50 \mathrm{~m} l$ of acetonitrile was added. Then the heterogeneous system was cooled to $-10^{\circ} \mathrm{C}$. A solution of NCA, $0.012 \mathrm{~mol}$, in acetonitrile was added to the cooled system and allowed to react for $2 \mathrm{hr}$ at $-10^{\circ} \mathrm{C}$ with stirring. After the reaction, the acetonitrile layer of the system was taken off and the aqueous layer was washed with $50 \mathrm{ml}$ of acetonitrile under cooling in order to remove unreacted NCA. The aqueous solution was warmed to $40^{\circ} \mathrm{C}$ for $5-10 \mathrm{~min}$, and was again cooled to $-10^{\circ} \mathrm{C}$ after addition of $30 \mathrm{~m} l$ of acetonitrile and $20 \mathrm{~m} l$ of water. The solution of another NCA in acetonitrile was added to the heterogeneous system and allowed to react by the same method as described above. After the sequential addition of NCA as above, the aqueous layer was washed with a mixture of ethyl acetate and acetonitrile- $(1: 1)$ and ethanol was added to the solution to remove sodium carbonate. Then the alcoholic solution was neutralized with dilute sulfuric acid or acetic acid and concentrated under reduced pressure at $35^{\circ} \mathrm{C}$. Crude peptide was obtained by addition of ethanol and diethyl ether. Recrystallization from water or TFA gave a pure peptide.

\section{NMR Measurement}

The high-resolution NMR spectra were obtained with a Varian A-60 spectrometer at room temperature. Tetramethylsilane was used as an internal standard. Concentration of the samples was adjusted to $10 \%$. 
Stepwise Synthesis of Oligopeptides with NCA. V.

Acknowledgement. The authors are indebted to Dr. F. Toda of the University of Tokyo for reading this manuscript and many useful suggestions. This work was supported by the Scientific Fund of the Ministry of Education.

\section{REFERENCES}

1. J. E. Shields, S. T. McDowell, J. Pavlos, and G. R. Gray, J. Amer. Chem. Soc., 90, 3549 (1968).

2. M. Goodman and E. E. Schmitt, ibid., 81, 5507 (1959).

3. M. Goodman, E. E. Schmitt, and D. A. Yphantis, ibid., 82, 3483 (1960).

4. M. Goodman, E. E. Schmitt, and D. A. Yphantis, ibid., 84, 1283 (1962).
5. M. Goodman and F. Boardman, ibid., 85, 2483 (1963).

6. M. Goodman and I. G. Rosen, Biopolymers, 2, 519 (1964).

7. M. Goodman and M. Langsman, ibid., 4, 275 (1966).

8. Y. Iwakura, K. Uno, M. Oya, and R. Katakai, ibid., 9, 1419 (1970).

9. R. Katakai, M. Oya, K. Uno, and Y. Iwakura, ibid., 10, 2199 (1972).

10. R. Katakai, M. Oya, K. Uno, and Y. Iwakura, J. Org. Chem., 37, 327 (1972).

11. M. Sheinblatî, J. Amer. Chem. Soc., 88, 2845 (1966).

12. F. A. Bovey and G. V. D. Tiers, ibid., 81, 2845 (1959). 